Journal of trends in Computer Science and Smart technology (TCSST) (2020)

Vol.02/ No. 02

Pages: 68- 77

https://www.irojournals.com/tcsst/

DOI: https://doi.org/10.36548/jtcsst.2020.2.001

\title{
Computer Vision on IOT Based Patient Preference Management System
}

\author{
A. Sathesh, \\ Department of EEE, \\ Eritrea Institute of Technology, \\ Eritrea. \\ Email: Sathesh4you@gmail.com
}

\begin{abstract}
Patient preference management is an essential work for any healthcare scheme to give priority to the needy patient. The work is generally carryout by a caretaker in the healthcare block to enroll their details of the patient on computer to find out and suggest an available consultant and time slot for the patient. These kind of usual works can be helpful up to certain normal conditions only. During uncertain times like viral explosion or war or nature disaster, the usual system will make the patient to wait in a queue for enrollment process. Most of the time it is intolerable to make a severe injured person to wait in the queue for the treatment. At the same time, during viral explosion the people were asked to stay at their home and for treatment they have to make a phone call to the care taking team for expressing their situation and health status. Attending a huge number of phone calls manually and providing a good suggestion to the caller is a challenging work for any healthcare team. The proposed IoT based computer vision system suggests the patient to send their status through a mobile phone message or email to the healthcare server to segregate the status of patient as emergency, severe and follow-up categories. This makes the healthcare team to identify the needy patient at right time to serve them. The proposed system is simulated with different computer vision algorithm and analyses its accuracy, time delay and drop rate to make a reliable patient preference management system.
\end{abstract}

Keywords: IoT data management, IoT patient management, big data energy consumption

\section{Introduction}

Viral disease spread control is a challenging work for any country. The primary task implemented by most of the nations is lockdown. The people of the countries were asked to stay at their home but the essential people of the healthcare team has to visit so many places in the infected areas to screen-out the basic health status of the common people. The basic health status like body temperature, throat pain, and their health history and sometimes even their travel history has to be noted for identifying the infected people and chance for people to be infected. The health status report of such huge data is difficult to analyze manually for prediction of infected person. A computer assisted system is generally used of sorting out the details of the data collected. But the proposed system avoids the healthcare team to visit the infected area and the data from those areas collected through an IoT based system. Similarly an artificial intelligence based computer

ISSN: 2582-4104 
Journal of trends in Computer Science and Smart technology (TCSST) (2020)

Vol.02/ No. 02

Pages: 68- 77

https://www.irojournals.com/tcsst/

DOI: https://doi.org/10.36548/jtcsst.2020.2.001

vision system is employed in the proposed scheme to manage such data for preparing the patient preference list to the healthcare team. This saves the time consumed for data collection and it avoids the questionable viral spread to the healthcare team visiting such infected areas.

The proposed patient preference system also analyses the existing patient report saved in the healthcare center server to find out the chances of people who may be get the viral infection. Each viral disease has its own nature in infection some of the disease infects the lungs, some of the disease may infect a person throat or eye or liver or some other organs in the human body. The proposed system identifies the patients with poor organs or recently infected organ people to alert and monitor their health status in such lockdown time. An IoT based system is needed for such operations to send alert and collected the necessary symptom data. Along with that a very big cloud space is needed to save all those collected data and a better computer vision based algorithm with higher accuracy and less computational time with lesser drop rate is needed achieving a better management system. Figure 1 shows the general architecture of IoT management system

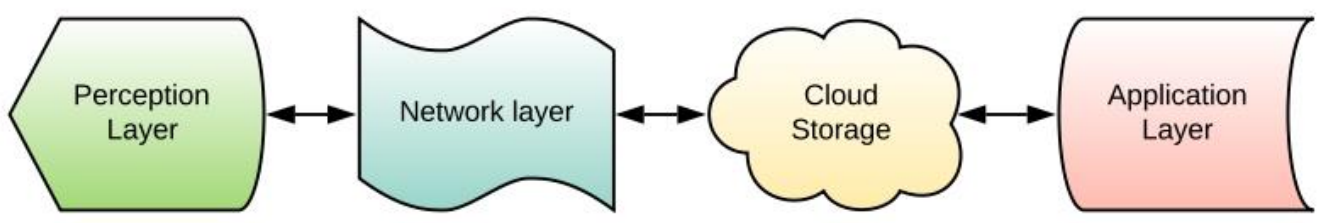

Figure 1. Basic architecture of IoT system

The IoT management system usually consists of perception layer, network layer, cloud layer and application layer for its operation. The perception layer is connected with required sensors and other tools for data collection process. The perception layer and network layer are mostly placed together for energy management. A single energy source will be placed between the layers to share the energy needed for its operation. The network layer transmits the collected data from the tools fixed in the perception layer to the cloud storage for data management. The network layer can be a mobile phone network, wifi network, intranet or a wired internet connection. The cloud storage stores the transmitted data in the allocated space for future operations with the collected data. The application layer can be any medium used to fetch the stored data for management analysis and operations. There are several algorithms and tools were introduced to analyze the data located in the cloud storage, few of them are discussed in the following section.

ISSN: 2582-4104 
Journal of trends in Computer Science and Smart technology (TCSST) (2020)

Vol.02/ No. 02

Pages: 68- 77

https://www.irojournals.com/tcsst/

DOI: https://doi.org/10.36548/jtcsst.2020.2.001

\section{Related work}

There are several techniques were introduced to manage the data collection and data transmission in IoT technology to make it reliable and fast in nature. A non-linear closed loop control system [1] was proposed to improve the efficiency of IoT management system. The closed loop adaptive algorithm increases the searching speed of the data stored in the management system. The queuing theory is enforced here to make the data in to miner blocks for providing easy search option to the algorithm. Along with queuing theory, hash maps were also added here to improve the system reliability. A secure and smart framework for hospital management using IoT and artificial intelligence [2] was introduced to manage the patient data, patient monitoring, treatment and diagnosis framework. The secure framework secures the collected data save in cloud to avoid manipulation and hackers attack. The limitations in computation time, memory available, energy consumption were also discussed in this work to make a reliable framework.

A green IoT based architecture [3] was framed to manage the agriculture field with self-energy generation for supplying the transmission nodes and cloud management system. The green IoT may reduce the cost of installation and maintenance cost than the traditional IoT architecture. The architecture is developed to work continuously for its life time in managing the planting field. This improves the growth cycle of the plantation product. A minimized cloud computing model based on edge computing with artificial intelligence technique [4] was structured to minimize the time consumption for cloud computing data. The data which are used continuously and frequently will be moved to offloaded cloud for accessing it in lesser time. The data in the offloaded cloud has lesser space with lesser data. It will be help full in taking the frequent data faster than taking it from the edge cloud space. The data to be stored in the offloaded cloud is managed by an artificial intelligence algorithm framed in the IoT structure. The work is simulated with network simulator for performance evaluation and it shows minimal delay with more number of tasks than the other techniques. An IoT energy platform [5] was developed to manage the energy consumption on IoT platform. The platform procedure can be used for analyzing the performance of IoT based data transmission. By managing the energy requirement for transmission makes the system to run without power failure and other nature.

An improved flow and mobility management system [6] was implemented to confirm the flow process of data in IoT network in systematic manner. The management system manages the scheduling time for the data to transmit from source to destination point for improving the success rate. So that the cloud can also save the data systematically without losing the information in time to time. The mobility network manages the multiple data arrival to the system in by rescheduling its receiving time. A disaster management system based on IoT technology [7] is provided for planning the rescue operation during natural disaster situation. A task technology fit algorithm is added for verifying the technology performance in disaster management. The distributed block chain involved trust system [8] was arranged to manage the IoT infrastructure in

ISSN: 2582-4104 
Journal of trends in Computer Science and Smart technology (TCSST) (2020)

Vol.02/ No. 02

Pages: 68- 77

https://www.irojournals.com/tcsst/

DOI: https://doi.org/10.36548/jtcsst.2020.2.001

block chain module. The system defines the connected node as separate entities to transmit the data in an efficient manner to the cloud space. The allocation of memory space in the cloud can also be desired by this architecture to form a uniform data movement between the station and storage point. The monitoring devices and mass storage continuous devices are tagged separately for heavy storage and computation time.

A decentralized technique for IoT data management [9] is combined with the general data storage system. The IoT structure prepared by blockchain module is executed here for make the performance faster and better than the usual IoT data structures. The data are separated in to different category for faster retrieval and editing utilization. A block chain based IoT system [10] is developed to secure the data stored in the transport layer. As the data are moving from different scenarios, it is possible for the data to be lost in between or stolen by the hackers. There are also chances for missing few data during the transmission. But the clock chain enabled IoT system has the ability to secure the data efficiently than the other methods. The data in the IoT systems are decentralized for faster data exchange and at the same time data security is also easy here to implement. Securing the data into small group as blokchain makes the work easier. The fog nodes in cloud system [11] is enforced to access the stored data in online and offline modes. The processing of data is made easier when applying it with fog nodes. The fog nodes are interconnected to each other for data transparency and classification analysis. A treatment planning data analysis for patients in IoT system [12] is established for extracting the necessary information from cloud storage unit. The cloud storage is communicated with more number of sensors and their nodes for receiving continuous signals. These signals produces a huge traffic and the system reliability as a questionable one. The system makes the cloud storage process with lesser traffic by screening the data moving between the cloud and the collector side. By screening the data the amount of data moving towards the cloud can be controlled for saving the energy and cloud storage.

A smart farm data analysis IoT system [13] is implemented to manage the collected information about the soil and climate content. The data collected by the help of sensors are gathered in the cloud system to manipulate the conditions about the farming field. The data in the cloud storage is also accessible from a mobile application to control the farming field irrigation system. The cloud data is stored for analyzing the performance of the farming field in climatic changes to suggest farming ideas intelligently. A cloud fog based big data analysis method [14] was presented to analyze the collected data from various resources for data management. The collected information are verified in the fog layer to move the necessary information to its correct destinations without saving it to the cloud storage. The cloud storage data can be transmitted from the fog layer as well as the destination manager for making the storage space in efficient manner. The data to be saved in the cloud system is also scheduled in the fog layer to avoid the traffic in data movement. The ant colony optimization with multipath distance algorithm [15] is suggested to improve the power efficiency in IoT systems. The data accessed through multipath system avoids the data drop in transmission and it saves the energy requirement for resending the data to the destination station. The multipath system

ISSN: 2582-4104 
Journal of trends in Computer Science and Smart technology (TCSST) (2020)

Vol.02/ No. 02

Pages: 68- 77

https://www.irojournals.com/tcsst/

DOI: https://doi.org/10.36548/jtcsst.2020.2.001

also avoid the delay in data transmission, as the data are forwarded through the vacant path to reach the destination. A machine learning approach for cloud data management [16] is developed to manage the storage allocation in cloud. The machine learning approach makes the system performance better in allocating the memory space. The cloud resource utilization is analyzed with network simulator tool and found the performance is satisfied than the usual storage management system.

\section{Proposed Work}

The proposed patient preference management system is an improved IoT module added with artificial intelligence structure for moving the data to the cloud and application layer efficiently. Figure 2. Indicates the blocks involved in the proposed patient management system. The system differs from the usual IoT system in terms of adding additional artificial intelligence layer between the network layer and cloud layer termed as primary AI screening layer and the secondary AI screening layer is placed between application layer and fog layer. The fog layer acts a temporary storage unit comparatively lesser space than the cloud storage for faster movement in data which are required frequently by the application layer or perception layer. The secondary AI layer maintains the work on segregate the data to be stored in fog layer and cloud layer in the IoT system. The primary AI layer is employed to move the most urgent data to the application layer for alerting purpose. The received data in the application layer can be stored either in the fog layer or cloud storage with respect to the decision taken by the application layer and the secondary AI layer.

The primary AI screening layer is also connected with both cloud storage for storing the non-emergency collected data without application layer decision. These data can be managed by the application layer directly by the secondary AI layer. The proposed system is analyzed with several computer vision algorithm in the AI screening layers for detecting the performance of the proposed system. A flexible fuzzy regression algorithm is activated in the proposed system for managing the data between cloud storage and application layer. The Fuzzy Regression Relationship (FRR) is expressed by the following equation.

$$
\hat{f}=x_{0} y_{0}+x_{1} y_{1}+\cdots+x_{k} y_{k}
$$

The (y) variables are independent and (f) variables are dependent with a fuzzy number. But in case if the (y) variables are fuzzy numbered with independent variable then the (f) is also a dependent fuzzy number. The (k) values are assumed as fuzzy number on symmetric values estimated in regular intervals.

ISSN: 2582-4104 
Journal of trends in Computer Science and Smart technology (TCSST) (2020)

Vol.02/ No. 02

Pages: 68- 77

https://www.irojournals.com/tcsst/

DOI: https://doi.org/10.36548/jtcsst.2020.2.001

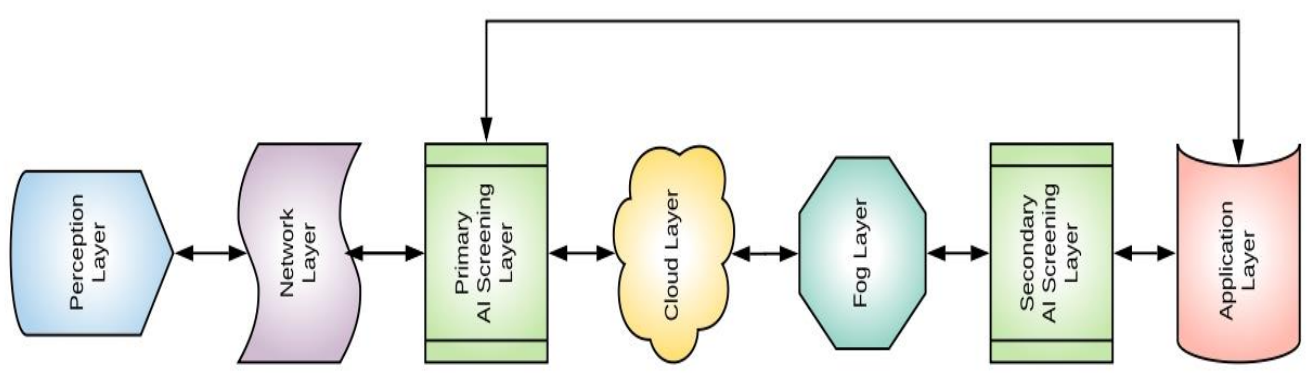

Figure 2. Architecture of the proposed patient management system.

A Multi Algorithm Service (MAS) model is also fixed in the system to find out the performance of the proposed system. The processing model of the service model algorithm is defined as $M_{1}$. In the process $\mathrm{x}, \mathrm{y}$ is represented as process speed. The weight factor of the data content is mentioned as $\mathrm{W}_{\mathrm{k} .}$ similarly $\mathrm{t}$, $\mathrm{f}, \mathrm{g}$ are the data transmitted as different time.

$$
M_{1}=\min _{x, y} \sum_{k=1}^{k} \frac{1}{w_{k}}\left[t_{k} f_{k}+\left(1-t_{k}\right) g_{k}\right]
$$

An Adaptive Wavelet Sampling (AWS) method was also performed in the proposed system for analysis. The AWS algorithm is developed for text data management by saving the energy requirement for the transmitting nodes. The text data management is formulated by sampling the transmitted data in to several pieces with respect to time ( $\mathrm{t}$ ). It is helpful in transmitting the emerging nodes first to the application layer. The equation of AWS is expressed as,

$$
\rho_{t}=h\left(\frac{\text { tex }_{\text {total }}}{\text { tex }_{\text {con }}}\right)
$$

The total amount of data transmitted with respect to time $\rho_{t}$ is expressed as total amount of data available for transmission to the content of the text data in the transmission process. The sampling rate sequence $\left(G_{S}\right)$ is expressed as combination of environmental parameters and the noise absorption of the transmission layer.

ISSN: 2582-4104 
Journal of trends in Computer Science and Smart technology (TCSST) (2020)

Vol.02/ No. 02

Pages: 68- 77

https://www.irojournals.com/tcsst/

DOI: https://doi.org/10.36548/jtcsst.2020.2.001

$$
G_{s}=G_{s e q}+G_{n}
$$

An Outliner Detection Algorithm (ODA) is also analyzed with the proposed patient management system. A canopy algorithm is added in this formulation for clustering the data model in the beginning signal. Then a K-means algorithm comes next to the canopy for further model data of the text combinations. The Kmeans algorithm predicts the size of the outliner pockets of transmission. The centroid space and distance are calculated by Euclidean instance distance factor. If the distance of the pockets are too high it formulate an outliner to the data.

The semantic layer is the opening layer of the ODA algorithm utilized to manage the service occurs in the process of algorithm. Tex textual content of the data transmission are gathered here for its operation module. The pattern identifier is implemented to classify the data text to be transmitted in to several category for ease of recognition. The recognized data texts are formulated in to their respect group for data transmission and it is helpful in estimate the amount of data to be transmitted with required energy for transmission. Follow to the serviceable layer the service monitor is fixed for scheduling the process transmission. The scheduled data are waiting here in the temporary storage unit for some time to make up the movement event of the node pockets. The devices blocks which are connected in the proposed management systems are commonly managed by the service monitor of the ODA algorithm. The network layer is connected towards the service monitor for transmitting the scheduled node pockets to its destination remotely. The data from the semantic layer to the network layer are secured with an encryption algorithm for protecting the data.

\section{Results and Discussion}

The proposed computer vision patient preference system is verified with several AI based algorithm to identify a suitable algorithm for managing the records and data efficiently. The performance is evaluated with success rate with respect to number of user requests transmitted from the perception layer. Similarly the performance is evaluated with the system speed. The execution time of the proposed system with various algorithm is analyzed to tabulate the performance comparison between the algorithms. Power consumption of the different algorithm is calculated in the proposed approach for identifying the system energy requirements.

The throughput of the proposed system with FRR, MAS, AWS and ODA algorithm is calculated for number of data request transferred with successful delivery at different time is analyzed and graphed in the figure 3. The power requirement for transmitting the data from source to destination is calculated for various number of data request for finding the efficiency of the proposed system. The execution time is

ISSN: 2582-4104 
Journal of trends in Computer Science and Smart technology (TCSST) (2020)

Vol.02/ No. 02

Pages: 68- 77

https://www.irojournals.com/tcsst/

DOI: https://doi.org/10.36548/jtcsst.2020.2.001

measured for each data request to find the execution speed of different algorithms insisted in the proposed system.

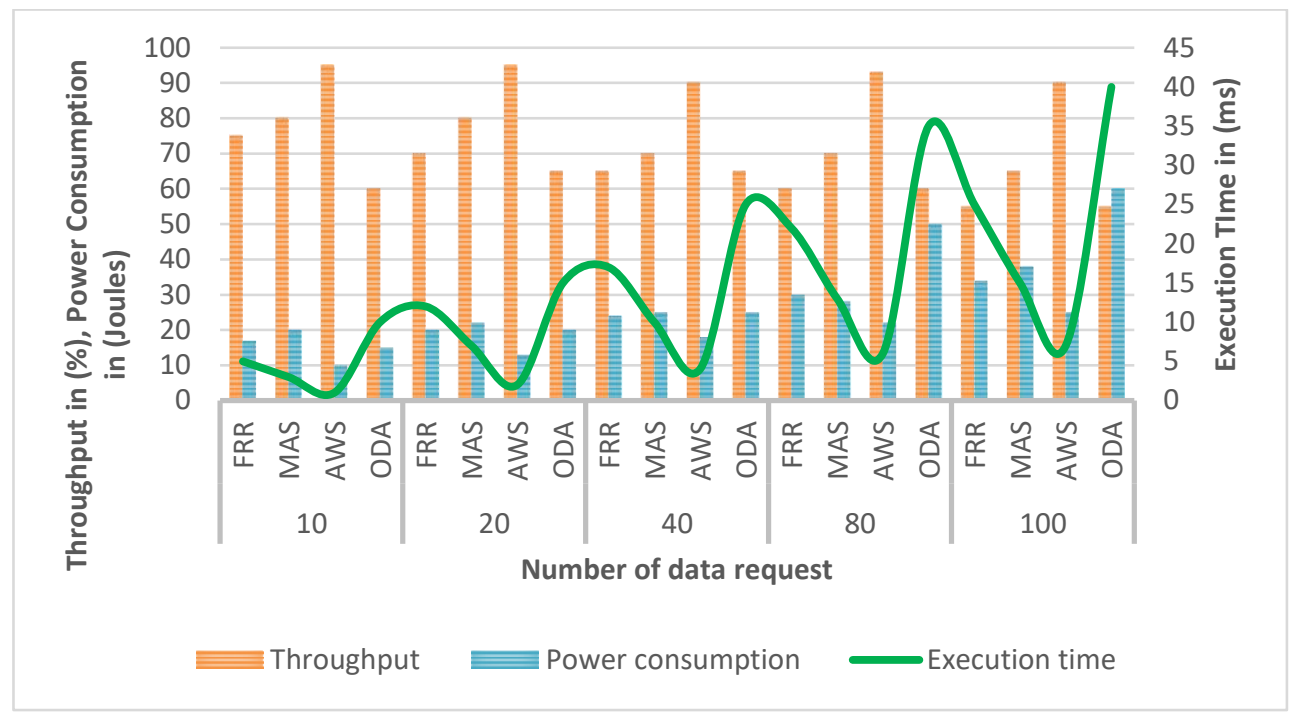

Figure 3. Performance of the proposed patient preference management system.

The performance of the AWS algorithm is comparatively better than the FRR, MAS and ODA algorithm. The throughput of the AWS algorithm performance well in all the scenario of data requests. The ODA algorithm throughput is increased with respect to the number of data requests. But the performance is FRR and MAS is decreased with respect to number of data requests. The AWS algorithm also performs similar to the FRR and MAS algorithm where the through put is gradually reduced when number of data request in increased. The power consumption of the AWS algorithm is comparatively works well than the other algorithm with reduced energy consumption for data transmission. The ODA algorithm power consumption is increased during higher data requests. Similarly, the execution time of the AWS algorithm did good performance and increased slightly when data request is increased.

\section{Conclusion}

The proposed computer vision based patient preference management system is performed with different algorithm for identifying its performance in data transmission. The same amount of data is repeatedly tested with the existing algorithms for finding their performance in data transmission and management from the

ISSN: 2582-4104 
Journal of trends in Computer Science and Smart technology (TCSST) (2020)

Vol.02/ No. 02

Pages: 68- 77

https://www.irojournals.com/tcsst/

DOI: https://doi.org/10.36548/jtcsst.2020.2.001

perception layer to the application layer. The analysis result indicates that the AWS algorithm with the proposed architecture performs better than the other algorithms. So that the AWS algorithm can be employed as a computer vision manager for segregating the required data to move between the application layer and cloud storage. This improves the system throughput and minimizes the power consumption and execution time of the data movement.

\section{References}

[1] Casado-Vara, Roberto, Pablo Chamoso, Fernando De la Prieta, Javier Prieto, and Juan M. Corchado. "Non-linear adaptive closed-loop control system for improved efficiency in IoTblockchain management." Information Fusion 49 (2019): 227-239.

[2] Valanarasu, Mr R. "Smart and secure IoT and AI integration framework for hospital environment." Journal of ISMAC 1, no. 03 (2019): 172-179.

[3] Ruan, Junhu, Yuxuan Wang, Felix Tung Sun Chan, Xiangpei Hu, Minjuan Zhao, Fangwei Zhu, Baofeng Shi, Yan Shi, and Fan Lin. "A life cycle framework of green IoT-based agriculture and its finance, operation, and management issues." IEEE communications magazine 57, no. 3 (2019): 90-96.

[4] Pandian, A. Pasumpon. "Enhanced edge model for big data in the internet of things based applications." Journal of trends in Computer Science and Smart technology (TCSST) 1, no. 01 (2019): 63-73.

[5] Terroso-Saenz, Fernando, Aurora González-Vidal, Alfonso P. Ramallo-González, and Antonio F. Skarmeta. "An open IoT platform for the management and analysis of energy data." Future Generation Computer Systems 92 (2019): 1066-1079.

[6] Pandian, M. Durai. "Enhanced network performance and mobility management of IoT multi networks." Journal of trends in Computer Science and Smart technology (TCSST) 1, no. 02 (2019): 95-105.

[7] Sinha, Akash, Prabhat Kumar, Nripendra P. Rana, Rubina Islam, and Yogesh K. Dwivedi. "Impact of internet of things (IoT) in disaster management: a task-technology fit perspective." Annals of Operations Research 283, no. 1-2 (2019): 759-794.

[8] Haoxiang, Wang. "Trust management of communication architectures of internet of things." Journal of trends in Computer Science and Smart technology (TCSST) 1, no. 02 (2019): 121-130.

[9] Xiong, Zehui, Yang Zhang, Nguyen Cong Luong, Dusit Niyato, Ping Wang, and Nadra Guizani. "The best of both worlds: A general architecture for data management in blockchainenabled Internet-of-Things." IEEE Network 34, no. 1 (2020): 166-173.

[10] Sivaganesan, D. "Block chain enabled internet of things." Journal of Information Technology 1, no. 01 (2019): 1-8.

ISSN: 2582-4104 
Journal of trends in Computer Science and Smart technology (TCSST) (2020)

Vol.02/ No. 02

Pages: 68- 77

https://www.irojournals.com/tcsst/

DOI: https://doi.org/10.36548/jtcsst.2020.2.001

[11] Zeng, Wenxi, Shuai Zhang, I-Ling Yen, and Farokh Bastani. "Semantic IoT Data Description and Discovery in the IoT-Edge-Fog-Cloud Infrastructure." In 2019 IEEE International Conference on Service-Oriented System Engineering (SOSE), pp. 106-10609. IEEE, 2019.

[12] Smys, S., and Jennifer S. Raj. "Internet of things and big data analytics for health care with cloud computing." Journal of Information Technology 1, no. 01 (2019): 9-18.

[13] Muangprathub, Jirapond, Nathaphon Boonnam, Siriwan Kajornkasirat, Narongsak Lekbangpong, Apirat Wanichsombat, and Pichetwut Nillaor. "IoT and agriculture data analysis for smart farm." Computers and electronics in agriculture 156 (2019): 467-474.

[14] Bestak, Robert, and S. Smys. "Big data analytics for smart cloud-fog based applications." Journal of trends in Computer Science and Smart technology (TCSST) 1, no. 02 (2019): 74-83.

[15] Krishnaraj, N., and S. Smys. "A Multihoming ACO-MDV Routing for Maximum Power Efficiency in an IoT Environment." Wireless Personal Communications 109, no. 1 (2019): 243-256.

[16] Chandy, Abraham. "Smart resource usage prediction using cloud computing for massive data processing systems." Journal of Information Technology 1, no. 02 (2019): 108-118.

Biography: Prof .Sathish works in the Department of EEE, in Eritrea Institute of Technology, Eritrea. His interested area of research includes modern computer Science and emerging smart technologies in various aspects of Wireless Communications, Cloud Computing, Computer System Engineering, Communication Technologies, Information Processing, Computer Networks, Web Technologies, Computing \& Communications, Automation, Image processing and Wireless Communications.

ISSN: 2582-4104 